\title{
P101 Arterial Stiffness vs Sarcopenia in Portuguese Elderly Population
}

\author{
Valentina Vassilenko ${ }^{1,4,5}$, Andreia Serrano ${ }^{1,4,5}$, Beatriz Ramalho ${ }^{1}$, Paulo Bonifácio ${ }^{1,4,5}$, Ana C. Coelho ${ }^{2}$ \\ Fernando Pimentel-Santos ${ }^{3}$ \\ ${ }^{1}$ Laboratory of Instrumentation, Biomedical Engineering and Radiation Physics (LIBPHYS), NOVA School of Science and \\ Technology - NOVA University Lisbon, 2829-516 Caparica, Portugal \\ ${ }^{2}$ Santa Casa da Misericórdia de Almada, Portugal \\ ${ }^{3}$ Centre for Chronic Diseases, Nova Medical School (CEDOC|FCM- UNL), Lisbon, Portugal \\ ${ }^{4} N M T$, S.A., Parque Tecnológico de Cantanhede, Núcleo 04, Portugal \\ ${ }^{5}$ Iberian Network on Central Hemodynamic and Arterial Structure, Lisbon, Portugal
}

\begin{abstract}
Aging often results in the appearance of sarcopenia, which is characterized by loss of muscle mass and strength. The incidence of sarcopenia in the elderly is a quite common and leads to weakness and disability, as well as a lower quality of life, also assuming a high social and economic impact. One previous study has shown an independent negative association between arterial stiffness and skeletal muscle mass decline [1], which suggest its relationship with sarcopenia. This work aims to evaluate the relation between sarcopenia and the arterial stiffness, in Portuguese elderly population A cohort of 38 elderly volunteers between 67 and 95 years, with and without Sarcopenia, were selected from nursing homes and day centers of Portuguese charity institution Santa Casa da Misericórdia de Almada. All volunteers have the same living condition, meals and lifestyle routine, excepting the medications. The diagnosis and degree (severe or moderate) of sarcopenia were performed according to the European consensus on definition and diagnosis of sarcopenia [2] at the same day with arterial stiffnesses measurements assessed by carotid-femoral Pulse Wave Velocity (cfPWV). Our data show a positive correlation for cfPWV with the sarcopenia severity degree, which represents an increasing in arterial stiffness and might be explained by the fact that the loss of muscle mass is often associated with chronic inflammation [3]. Despite of the small cohort size, the male gender held a bigger mean value of cfPWV, with significant $p$-value (0.014), in comparison to the female gender, which estimates a higher CV risk for the male elders of the region of Almada and Greater Lisbon compared to the female gender. Further investigations would be desirable in order to obtain larger samples and ascertain the estimates for the respective groups.
\end{abstract}

\section{REFERENCES}

[1] Abbatecola AM, Chiodini P, Gallo C, Lakatta E, Sutton-Tyrrell K, Tylavsky FA, et al. Pulse wave velocity is associated with muscle mass decline: Health ABC study. Age (Dordr) 2012;34:469-78.

[2] Cruz-Jentoft AJ, Baeyens JP, Bauer JM, Boirie Y, Cederholm T, Landi F, et al. Sarcopenia: European consensus on definition and diagnosis: Report of the European Working Group on Sarcopenia in Older People. Age Ageing 2010;39:412-23.

[3] Rodríguez AJ, Karim MN, Srikanth V, Ebeling PR, Scott D. Lower muscle tissue is associated with higher pulse wave velocity: a systematic review and meta-analysis of observational study data. Clin Exp Pharmacol Physiol 2017;44:980-92.

(c) 2019 Association for Research into Arterial Structure and Physiology. Publishing services by Atlantis Press International B.V. This is an open access article distributed under the CC BY-NC 4.0 license (http://creativecommons.org/licenses/by-nc/4.0/). 